Título artículo / Títol article: $\quad$ Amorphous Iron Oxyhydroxide Nanosheets:

Synthesis, Li Storage, and Conversion Reaction Kinetics

Autores / Autors

Xu, Chen ; Zeng, Yi ; Rui, Xianhong ; Zhu, Jixin ; Tan, Huiteng ; Guerrero Castillejo, Antonio ; Toribio, Juan ; Bisquert, Juan ; García Belmonte, Germà ; Yan, Qingyu

Revista:

The Journal of Physical Chemistry C (2013) vol. 117, no 34

Versión / Versió:

Preprint

Cita bibliográfica / Cita

XU, Chen, et al. Amorphous Iron Oxyhydroxide bibliogràfica (ISO 690):

Nanosheets: Synthesis, Li Storage, and Conversion Reaction Kinetics. The Journal of Physical Chemistry C, 2013, vol. 117, no 34, p. 17462-17469.

url Repositori UJI:

http://hdl.handle.net/10234/85070 


\title{
Amorphous Iron Oxyhydroxide Nanosheets: Synthesis, Li Storage and Conversion Reaction Kinetics
}

\author{
Chen Xu, ${ }^{a, b}$ Yi Zeng, ${ }^{a}$ Xianhong Rui, ${ }^{a}$ Jixin Zhu, ${ }^{b}$ Huiteng Tan, ${ }^{a,}$ Antonio Guerrero, ${ }^{c}$ \\ Juan Toribio, ${ }^{c}$ Juan Bisquert, ${ }^{c}$ Germà Garcia-Belmonte, ${ }^{c, *}$ and Qingyu Yan ${ }^{a, b, *}$
}

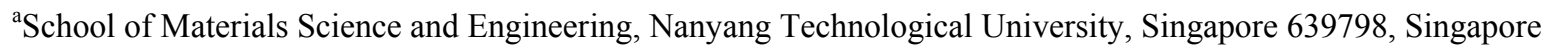

${ }^{\mathrm{b}}$ TUM CREATE Research Centre@NTU, Nanyang Technological University, Singapore 637459, Singapore

${ }^{\mathrm{c} P h o t o v o l t a i c}$ and Optoelectronic Devices Group, Departament de Física, Universitat Jaume I, 12071 Castelló, Spain

\section{Corresponding Authors}

"Qingyu Yan E-mail: alexyan@ntu.edu.sg

*Germà Garcia-Belmonte E-mail: garciag@fca.uji.es

\begin{abstract}
We present a facile approach to synthesize amorphous iron oxyhydroxide nanosheet from the surfactant-assisted oxidation of iron sulphide nanosheet. The amorphous iron oxyhydroxide nanosheet is porous and has a high surface area of $223 \mathrm{~m}^{2} \mathrm{~g}^{-1}$. The lithium storage properties of the amorphous iron oxyhydroxide are tested: it is a conversion-reaction electrode material, and it demonstrates superior rate capabilities (e.g. discharge capacities as high as $642 \mathrm{mAhg}^{-1}$ is delivered at a current density of $2 \mathrm{C}$ ). In the impedance spectroscopy analysis, a $R C$ subcircuit is set up to model the conversion reaction process. And, the time constant of the $R C$ subcircuit reproduces the hysteresis in the discharge/charge voltage profile, indicating that the conversionreaction has a thermodynamic origin rather than a kinetic limitation.
\end{abstract}

Keywords: amorphous; nanosheet; conversion reaction; Impedance spectroscopy.

Published in J. Phys. Chem. C 2013, 117, 17462-17469 
Lithium ion battery (LIBs) is one of the most practical and promising energy storage devices.[1] Electrode materials with higher capacities, better rate capabilities and more durable cycling performance are desired for future LIBs. [1] Recently, nanostructuring has proven to be effective for the improvement of electrochemical property. [2-4] In LIBs, nanomaterials lead to increased electrode/electrolyte contact areas and shortened charge diffusion path, permitting batteries to operate at higher power.

Besides of nanostructuring, other approaches, such as forming loosely porous network [5] and compositing with a buffering media (e.g. graphene), [5] are also attractive. Using amorphous rather than crystalline phases is another effective approach to enhance the stress tolerance and in turn improve the cycling stability. It was demonstrated that amorphous $\mathrm{TiO}_{2}$ nanotubes possess higher capacities and better capacity retention than that of anatase-phased crystalline $\mathrm{TiO}_{2}$ with identical composition and morphology;[6] It was shown that the isotropic nature of amorphous $\mathrm{SnO}_{2}$ is advantageous in overcoming the electrochemical and mechanical degradation during cycling comparing with its crystalline counterpart.[7] Amorphous hierarchical porous germanium oxide was reported as a high-capacity anode with excellent cyclability in both half cells and full cells.[8] Different lithium storage properties of amorphous $\mathrm{MoO}_{2}$ was reported as compared to its crystalline counterpart, and lithium storage at structural defects in amorphous $\mathrm{MoO}_{2}$ was proposed.[9]

$\mathrm{Fe}_{2} \mathrm{O}_{3}, \mathrm{Fe}_{3} \mathrm{O}_{4}$ and $\mathrm{FeOOH}$ have attracted great interest in various applications due to their natural abundance and eco-friendliness. [10-13] Controlled synthesis of $\mathrm{Fe}_{2} \mathrm{O}_{3}$ and $\mathrm{Fe}_{3} \mathrm{O}_{4}$ nanostructures and their applications in LIBs have been intensively investigated.[14-16] Although the theoretical specific capacity of $\mathrm{FeOOH}$ is as high as $903 \mathrm{mAh} \mathrm{g}^{-1}$, there are few reports on $\mathrm{FeOOH}$ as LIB anode material. Moreover, the understanding of the kinetics of the charge transfer process in the conversion-based electrode materials is not fully clear and worth studying in detail.

Herein, we report a controlled synthetic approach to prepare FeOOH nanostructure of amorphous phase and explore its electrochemical properties as anode in LIBs. The as-synthesized amorphous iron oxyhydroxide nanosheets have lateral lengths of 100-200 nm. In addition, the iron oxyhydroxide nanosheets are highly porous with a BET surface area of $223 \mathrm{~m}^{2} \mathrm{~g}^{-1}$. When tested as anode materials in LIBs, the amorphous iron oxyhydroxide based electrode demonstrates excellent rate capabilities. Electrochemical impedance measurements are carried 
out to further examine the kinetic processes of the amorphous electrode materials. A new equivalent circuit model has been proposed that captures the discharging/charging capacitance and characteristic frequency to be the essential mechanism governing the conversion reaction process.

The amorphous iron oxyhydroxide of porous nanosheets structure was synthesized by oxidation of ultrathin iron sulphide (FeS) nanosheets by $\mathrm{H}_{2} \mathrm{O}_{2} / \mathrm{H}_{2} \mathrm{O}$ solution at room temperature. The solution-based synthesis of ultrathin iron sulphide has been reported previously. [17] A detailed synthetic protocol and characterization of the product can be found in the supporting information.

The amorphous nature of the synthesized product is demonstrated by the X-ray diffraction (XRD) pattern (Figure 1), which shows a broad hump in the 2theta range of $20^{\circ}-35^{\circ}$ without any characteristic peaks. In order to know the chemical composition and ensure the completeness of the oxidation process, X-ray photoelectron spectroscopy (XPS) was conducted to find out the oxidation state of Fe and O. The XPS scan profile (Figure S1) of the full range $(0-1300 \mathrm{eV})$ reveals the presence of mainly $\mathrm{Fe}, \mathrm{O}$ and $\mathrm{C}$, indicating complete reaction of iron sulphide and formation of iron oxides or iron hydroxides. The $2 p_{1 / 2}$ and $2 p_{3 / 2}$ photoelectrons at 711 and $724 \mathrm{eV}$ of the Fe 2p XPS data (Figure S2a) correspond to the reported values for $\mathrm{Fe}^{3+}$ in iron oxides or iron oxyhydroxide $[18,19]$, which confirms complete oxidation of $\mathrm{Fe}^{2+}$ to $\mathrm{Fe}^{3+}$. The XPS spectrum of oxygen can be convoluted to three distinct peaks, indicating the presence of oxygen (Figure S2b) of three different states. The two convoluted peaks at $530.10 \mathrm{eV}$ and $531.50 \mathrm{eV}$ can be assigned to $\mathrm{O}^{2-}$ and $\mathrm{OH}^{-}$in $\mathrm{FeOOH}$, respectively. $[18,19]$ The other peak at $533.4 \mathrm{eV}$ attributes to the oxygen in surface absorbed water. [19] In order to know the chemical bonds present in this amorphous compound, the sample was characterized by Fourier Transform Infrared Spectroscopy (FTIR) within the wavelength range of $400-4000 \mathrm{~cm}^{-1}$. Figure S3 displays the FTIR spectrum of the amorphous sample and compares its crystalline counterparts obtained by annealing the amorphous sample at $800^{\circ} \mathrm{C}$. The red powder obtained after annealing is $\alpha-\mathrm{Fe}_{2} \mathrm{O}_{3}$ as indicated by X-ray diffraction pattern (Figure S4). For both samples, broad adsorption bands at $3400 \mathrm{~cm}^{-1}$ and $1640 \mathrm{~cm}^{-1}$ can be ascribed to the stretching vibration of O-H and vibrations of adsorbed water molecules, respectively.[20] The crystalline $\alpha-\mathrm{Fe}_{2} \mathrm{O}_{3}$ shows evident adsorption bands at $465 \mathrm{~cm}^{-1}$ and $549 \mathrm{~cm}^{-1}$, which can be attributed to the bending and 
stretching of Fe-O bond, respectively.[21] However, the amorphous iron oxide shows only broad and weak adsorption band in the characteristic adsorption range of Fe-O bond. The absorption peaks at 471 and $686 \mathrm{~cm}^{-1}$ can be assigned to the $\mathrm{Fe}-\mathrm{O}$ vibrational modes in $\mathrm{FeOOH}$. And the $\mathrm{Fe}-$ O stretching vibration is responsible for the adsorption at around $618 \mathrm{~cm}^{-1}$ in FeOOH.[19, 20] Based on the results above, the synthesized product is identified to be Amorphous Iron $\left(\mathrm{Fe}^{3+}\right)$ oxyhydroxide (AIO).

Scanning Electron Microscopy (SEM) image (Figure 2a) and low-magnification Transmission Electron Microscopy (TEM) image (Figure 2b) of the amorphous product reveals loose and corrugated graphene-like 2D nanosheet structure, indicating no morphology and dimension change from its predecessor, iron sulphide nanosheets. [19] The sulphide-to-oxide conversion process was conducted in deionized water with the assistant of surfactant cetrimonium bromide $(\mathrm{CTAB})$. The iron sulfide nanosheets were synthesized in organic solvent (Oleyamine) and capped by organic surfactant. $\mathrm{CTAB}$, as a surfactant, in one hand, facilitate uniform dispersion of $\mathrm{FeS}$ nanosheets in water, and in the other hand, prevent agglomeration of nanosheets in the oxidation process (Supporting Information Scheme 1). From the HRTEM image (Figure 2c), each individual nanosheet is observed to consist of nanoparticles aggregated in porous form. The diameters of the very fine nanoparticles are smaller than $5 \mathrm{~nm}$. As depicted by the HRTEM image (Figure 2d), the atomic lattice on the surface of nanosheets lacks regular periodicity, which further confirms the amorphous nature of the synthesized product. Nitrogen absorption/desorption measurements of the porous determined a Brunauner-Emmett-Teller (BET) surface area of $233 \mathrm{~m}^{2} \mathrm{~g}^{-1}$ (Figure S5). The adsorption hysteresis does not show any limiting adsorption at high p/po ( $>0.7)$, which also implies the presence of slit-shaped pores given by the aggregates of loosely coherent very fine nanoparticles. [22] The highly porous nanostructure and high surface area are attractive for electrochemical applications.

Electrochemical characterization was conducted to investigate the anode performance of the amorphous iron oxyhydroxide nanosheet based on two-electrode coin-type cells with Li metal as counter electrode.[17] The electrode material comprises of $70 \mathrm{wt} \%$ iron oxyhydroxide nanosheet, $20 \mathrm{wt} \%$ single-wall CNT (SWCNT) and $10 \mathrm{wt} \%$ binder Polyacrylonitrile (PAN). Figure 3a 
shows the voltage profile of the AIO nanosheet electrode at a current density of $100 \mathrm{mAg}^{-1}$ between $0.05 \mathrm{~V}$ and $3.0 \mathrm{~V}\left(\mathrm{vs} . \mathrm{Li}^{+} / \mathrm{Li}\right)$ for the $1^{\text {st }}, 2^{\text {nd }}$ and $3^{\text {rd }}$ discharge/charge cycles. In the $1^{\text {st }}$ cycle discharge, the Li insertion involves three distinguishable stages: a shallow slope between $2.8 \mathrm{~V}$ and $1.5 \mathrm{~V}$ and two distinguishable plateaus at around $1.4 \mathrm{~V}$ and $0.75 \mathrm{~V}$, respectively. The specific discharge capacity counts to $437 \mathrm{mAhg}^{-1}$ when the AIO electrode is discharged to $0.95 \mathrm{~V}$, which corresponds to the incorporation of approximately $1.45 \mathrm{~mol} \mathrm{Li}$ ions per mol of AIO. Exsitu examination of the active material was carried out. TEM and HRTEM images of the discharged active material to $0.95 \mathrm{~V}$ (Figure S6a and S6b) demonstrate the retention of its nanosheet morphology and amorphous nature. It is assumed that the initial two stages only involves surface $\mathrm{Li}$ storage and insertion of $\mathrm{Li}^{+}$into the porous amorphous nanostructure.[23] The specific capacity of $914 \mathrm{mAhg}^{-1}$ corresponds to approximately insertion of $3 \mathrm{~mol} \mathrm{Li}$ per mol of AIO at the discharge potential of $0.5 \mathrm{~V}$, implying complete conversion reaction between $\mathrm{Li}^{+}$ and $\mathrm{AIO}$ with the formation of elemental $\mathrm{Fe}$ and $\mathrm{Li}_{2} \mathrm{O}\left(\alpha-\mathrm{FeOOH}+3 \mathrm{Li}^{+}+3 \mathrm{e}^{--} \leftrightarrow \mathrm{Fe}+\mathrm{LiOH}+\right.$ $\left.\mathrm{Li}_{2} \mathrm{O}\right)$.[20, 23] Based on this conversion reaction, the theoretical discharge capacity of iron oxyhydroxide is $903 \mathrm{mAhg}^{-1}$. Ex-situ TEM image (Figure S6c) of the active material at this discharged state shows that the structural network remains but nanosheet has been partially destroyed. High-magnification TEM observation (Figure S6d) reveals formation of very fine nanorods. The $1^{\text {st }}$ cycle discharge shows a total specific capacity of $1210 \mathrm{mAhg}^{-1}$, and the extra capacity can be attributed to surface solid-electrolyte interphase (SEI) layer formation.[24] The cycling performance of the AIO based electrode at varying current densities is plotted in Figure 5 b. It depicts $5^{\text {th }}$ cycle discharge capacities of $985,797,642,465$, and $270 \mathrm{mAhg}^{-1}$ at current densities of $0.1 \mathrm{C}, 0.5 \mathrm{C}, 1 \mathrm{C}, 2 \mathrm{C}$ and $5 \mathrm{C}$, respectively $\left(1 \mathrm{C}=903 \mathrm{mAg}^{-1}\right)$. After cycling at $5 \mathrm{C}$, the specific capacities of the AIO electrode can only recover back to 607 and $870 \mathrm{mAhg}^{-1}$ when the current density changes back to $1 \mathrm{C}$ and $0.1 \mathrm{C}$, respectively. The high porosity and large active surface area contributes to the high capacity of the AIO electrode, but also cause continuous surface side reactions and thus results in capacity fading upon cycling.[25, 26] The AIO electrode demonstrates higher capacities than crystalline iron oxyhydroxide at same current densities.[7, 20, 23, 27] Some of the research reports proposed that the improved rate capability of amorphous electrode material results from higher lithium ion mobility in its loose structure and large quantities of structural defects. [9, 28] However, for conversion-reaction type electrode materials, conversion reaction kinetics plays a more determinative role in electrode rate 
capabilities than $\mathrm{Li}^{+}$diffusion does. Therefore, the conversion reaction kinetics of the AIO electrode needs to be investigated.

Electrochemical impedance spectroscopy (EIS) is one of the non-destructive techniques to for investigation of LIB's performance.[29, 30] Nyquist plots derived from impedance measurement and proposed equivalent circuits are usually combined in analyzing of electrode process kinetics. [29] In recent research, EIS has been rigorously applied in explaining intercalation reactions, such as estimating charge transfer resistance and the diffusion coefficient of lithium ions in intercalation-type electrode materials. [31,32] However, EIS has seldom been employed to study conversion reaction process. The electrochemical impedance measurement is performed at the stabilized open circuit voltage at varying states of discharge and charge, with amplitude of $5 \mathrm{mV}$ and in the frequency range of $10^{5} \mathrm{~Hz}$ to $0.01 \mathrm{~Hz}$. Nyquist plots of the EIS analysis of AIO nanosheet electrode at different states of discharge (SOD) and states of charge (SOC) during its $5^{\text {th }}$ cycle are shown in Figure $4 \mathrm{a}$ and $4 \mathrm{~b}$, respectively. The $5^{\text {th }}$ cycle is selected for analysis because the conversion reaction are expected to be reversible and stabilized afterwards.

The Nyquist plots consist of three parts: the high-frequency semicircle (HFS), mediumfrequency semicircle (MFS) and inclined low-frequency line (LFL). The Nyquist plots vary with the SOD and SOC, and the MFS and LFL tend to overlap in this case. Some of the impedance features can be easily interpreted as originated from well-known processes. At high frequencies (HFS) a rather constant arc is observed that corresponds to the parallel connection between the double layer capacitance $C_{d l} \approx 45 \mu \mathrm{F}$ and surface charge transfer resistance $R_{c t} \approx 200 \Omega$. An additional series resistance accounts for the solution contribution $R_{S} \approx 8 \Omega$. The LFL signals the occurrence of a voltage-modulated charging process which gives rise to a capacitive behaviour. The interpretation relies on the so-called chemical capacitance which informs on the electrode ability of varying the concentration $c$ of reacted $\mathrm{Li}^{+}$upon application of a differential change in the chemical potential $\mu$ (directly assimilated to the electrode potential upon steady-state conditions as $\mu=q V$, where $q$ is the positive elementary charge)[33-35]

$$
C_{\mu}^{c}=q L \frac{d c}{d V}
$$


Here $L$ represents the electrode thickness. The analysis of the MFS is by far much more speculative. As inferred by examining the impedance plots in Figure 4 the kinetics of the charging/discharging mechanism is dominated by the resistive contribution arising from MFS in comparison with $R_{\mathrm{ct}}$. For intercalation or insertion compounds the limiting rate mechanism is the diffusion of $\mathrm{Li}$ ions inside the active material. In those materials the intermediate-frequency resistance arises from the transport of ionic species before reaching stable sites within the matrix. Diffusion of ions gives rise to distinctive impedance patterns characterized by Warburg-like responses as $Z \propto(i \omega)^{-1 / 2}$ (being $\omega$ the angular frequency, and $i=\sqrt{-1}$ ). In opposition to that impedance pattern, a well-defined arc is encountered at MFS for the electrodes studied here. Models based on spatially-restricted ion diffusion were proposed to account for intermediatefrequency arcs relaying on a distribution of diffusion lengths [36], or electronic transport limitations [37]. A commonly used approach stated the simultaneous occurrence of two-parallel diffusion paths of dissimilar lengths. However the amorphous iron oxyhydroxide electrodes analyzed in this work function by conversion reactions that entail an overall material rearrangement both of chemical and structural nature. These considerations lead us to regard the conversion reaction as the limiting-rate process of the change in electrode state of charge. The corresponding resistive element is then labelled as conversion-reaction resistance $R_{c r}$, and is connected in series with the previously introduced $C_{\mu}^{c}$. A simple model accounting for the impedance of the conversion reaction which explains the underlying meaning of the circuit elements is outlined as an Appendix. The model is able to capture the essential connection between $C_{\mu}^{c}$ and $R_{c r}$, and the concentration of reacted species and charging current, respectively. The proposed model assumes that transport processes do not significantly contribute to the dissipative mechanism represented by the resistance, and is based on the usually reported mechanisms of intercalation of $\mathrm{Li}^{+}$ions, and subsequent reaction producing phase separation. The circuit element in series $R_{c r}$ and $C_{\mu}$ allows us to calculate the conversion-reaction time scale as the product $R_{c r} C_{\mu}$. The reciprocal corresponds to the response frequency

$$
\omega_{c r}=\frac{1}{R_{c r} C_{\mu}}
$$


which establishes the reaction kinetic regime. The MFS appears as a consequence of the inclusion in parallel of an additional capacitive element of small value in comparison with $C_{\mu}^{c}$ (see Appendix). Such capacitance is interpreted here as originated by the chemical capacitance corresponding to intercalated (not having reacted) $\mathrm{Li}^{+}$ions $C_{\mu}^{n}$. An equivalent circuit model which gathers all the aforementioned mechanisms is shown in Figure 4c. This equivalent circuit produces excellent fitting with the Nyquist plots as observed in examining Figure $4 \mathrm{a}$ and $4 \mathrm{~b}$, both for charging and discharging polarization regimes.

Although conversion reaction involves insertion and release of charges, we propose here that the rate-limiting process is the reaction itself and not the ion transport (see Appendix for a complete derivation). It can be modelled by means of a discharge and charge process as a series $R C$ circuit. As explained previously the time required for complete change in charging state of a series $R C$ circuit is given by the product $R_{c r} C_{\mu}^{c}$. The larger the time constant, the longer time is required for discharging or charging to the target voltage. The electrical parameters at different SOD and SOC have also been calculated from fitting and displayed in Figure 5. It is observed that $C_{\mu}^{c}$ exhibits a high value of order $0.1 \mathrm{~F}$. For voltages in excess of $0.6 \mathrm{~V} C_{\mu}^{c}$ shows slight variations between $0.05-0.10 \mathrm{~F}$, and exhibits a small peak at $\sim 0.7 \mathrm{~V}$ in good correlation with the inverse derivative of the discharge voltage profile in Figure 3a. The conversion reaction resistance $R_{\mathrm{cr}}$ demonstrates similar trends (Figure $5 b$ ), but exhibits much larger values for high voltages $(>2$ $\mathrm{V})$. This entails that the conversion reaction is hindered at low states of charge, but favoured as the ion concentration increases within the electrode. An averaged value $R_{c r} \approx 500 \Omega$ is encountered greater than $R_{c t}$. It is evidenced that the intermediate capacitance $C_{\mu}^{n}$ related to intercalated $\mathrm{Li}^{+}$ion concentration (Figure 4c) shows values two orders of magnitude below $C_{\mu}^{c}$, which accounts for concentration of reacted species The combination of $C_{\mu}^{c}, R_{c r}$, and $C_{\mu}^{n}$ forms a sort of relaxation subcircuit [38] which is interpreted here in terms of the conversion reaction mechanism. 
The conversion reaction kinetics can be alternatively accessed by examining the response frequency in Eq. (2). Values of $\omega_{c r}$ within the interval $0.3-0.6 \mathrm{~s}^{-1}$ are found. One can observe that $\omega_{c r}$ exhibits a maximum (marked with an arrow in Figure 5d), which is located within the potential interval of the major conversion reaction $\left(\mathrm{FeOOH}+3 \mathrm{Li}^{+}+3 \mathrm{e}^{-} \rightarrow \mathrm{Fe}+\mathrm{Li}_{2} \mathrm{O}\right.$ and $\left.\mathrm{LiOH}\right)$ according to the discharge voltage profile (Figure3a). We notice that a voltage shift in $\omega_{c r}$ of $\sim 0.5 \mathrm{~V}$ occurs between discharge and charge regimes. This fact points to the hysteretic behaviour of the conversion reaction [39, 40]. We highlight that EIS analysis reproduces the hysteresis observed between charge and discharge profiles (Figure 3a), or cyclic voltammetry. It can be inferred accordingly that the conversion reaction hysteresis has an origin related to intrinsic thermodynamics rather than to transport limitations.

In summary, amorphous iron oxyhydroxide nanosheet has been synthesized via surfactantassisted oxidation of the iron sulphide nanosheet. The highly porous nanostructure and high surface area of the amorphous iron oxyhydroxide nanosheet are attractive for electrochemical applications. When characterized as anode material in $\mathrm{LIB}$, it has conversion reaction with $\mathrm{Li}^{+}$ and demonstrates superior rate capability (e.g. discharge capacity as high as $465 \mathrm{mAhg}^{-1}$ is delivered at a current density of $2 \mathrm{C}$ ). A new equivalent circuit that directly models the conversion reaction process was set up by fitting the Nyquist plots from EIS analysis. The conversion reaction subcircuit (series $R C$ ) reproduces the hysteresis in the discharge/charge voltage profile, indicating that the conversion reaction has a thermodynamic origin rather than a kinetic limitation.

\section{Appendix}

In the following we outline a simple model accounting for the equivalent circuit of the conversion reaction mechanism. Let us express a conversion reaction as

$$
\mathrm{Li}^{+}+\mathrm{MX}+\mathrm{e}^{-} \rightarrow \mathrm{M}+\mathrm{LiX}
$$

where $\mathrm{Li}^{+}$is a lithium ion, $\mathrm{M}$ is a transition metal, and $\mathrm{X}=\mathrm{O}, \mathrm{S}, \mathrm{F} \ldots$ The stoichiometry is obviated for simplicity. It has been widely recognized that the voltage dependence on charge for 
systems undergoing a conversion reaction can be divided in several parts. [41, 42] In a first stage $\mathrm{Li}$ atoms enter the MX lattice in a usual intercalation process, with a marked step of the voltage. Thereafter the reaction of Eq. (3) proceeds producing phase segregation. In order to obtain a physical interpretation for the results of impedance spectroscopy measurements we outline here a simple model that incorporates the two mentioned stages. This model is based on a well known two state model that has been often used for describing intercalation systems with different lattice sites, and even including order-disorder transitions.[43-45] This model is rather crude for the present system, however it is rather illustrative of the fact that measured capacitances are not of interfacial origin. Since the model obeys detailed balance it incorporates time reversal properties, and the voltage hysteresis observed in conversion reactions cannot be described by this simple model.[40] It would be necessary to modify the kinetic coefficients in the model, which lies beyond the scope of the present work.

We assume that the MX lattice contains $N_{0}$ sites where $\mathrm{Li}^{+}$ions can diffuse and a total of $N_{1}$ atoms of type $\mathrm{X}$ per unit volume. $n \leq N_{0}$ is the concentration of diffusing ions in the lattice with energy $E_{0}$ (we assume $n<<N_{0}$ ) and $c \leq N_{1}$ is the concentration of $\mathrm{Li}^{+}$that have reacted to $\mathrm{LiX}$ with energy $E_{1}$. The equilibrium chemical potential of $\mathrm{Li}$ ions is

$$
\mu=\mathrm{E}_{0}+k_{B} T \ln \left(\frac{n}{N_{0}}\right)=E_{1}+k_{B} T \ln \left(\frac{c}{N_{1}-c}\right)
$$

It can be related to the electrode potential as $\mu=q V$ where $q$ is the positive elementary charge. Any change of voltage changes the concentration of Li ions in the LiMX particle. The mobile $\mathrm{Li}^{+}$ concentration at the surface relates to the voltage as

$$
n=N_{0} e^{-\left(E_{0}-q V\right) / k_{B} T}
$$

$\mathrm{Li}$ ions enter the particle, diffuse and react. This process is described by the kinetic equations

$$
\begin{aligned}
& \frac{\partial n}{\partial t}=-\frac{\partial J_{n}}{\partial x}-k_{1} n\left(N_{1}-c\right)+k_{2} c\left(N_{0}-n\right) \\
& \frac{\partial c}{\partial t}=k_{1} n\left(N_{1}-c\right)-k_{2} c\left(N_{0}-n\right)
\end{aligned}
$$

Here $J_{n}$ is the flux at position $x$, and $k_{1,2}$ correspond to the kinetic constants. For simplicity we consider a 1 dimensional system of thickness $L$ such that the injected current is $j=q J_{n}(0)$ and $J_{n}(L)=0$. We can also assume that diffusion in the lattice is quite fast, with respect to the reaction kinetics. This means that $n$ and $c$ are homogeneous, so that we can integrate Eq. (6) with the result

$$
\frac{\partial n}{\partial t}=\frac{J_{n}(0)}{L}-k_{1} n\left(N_{1}-c\right)+k_{2} c\left(N_{0}-n\right)
$$


The impedance of diffusion in this model has been described in Refs. [45-47]. In equilibrium the current is zero and we obtain

$$
\frac{k_{1}}{k_{2}}=\frac{N_{0} \bar{c}}{\bar{n}\left(N_{1}-\bar{c}\right)}
$$

Since we assume that $k_{1}$ and $k_{2}$ are constant the detailed balance condition is

$$
\frac{k_{1}}{k_{2}}=e^{\left(E_{0}-E_{1}\right) / k_{B} T}
$$

In transient or voltage modulated conditions the concentration of $n$ is modified by the external potential and $c$ will change towards the equilibrium value indicated in Eq. (4). For a modulation of the density $n=\bar{n}+\hat{n}$, we find by Eq. (5) that the free ion density modulation depends on voltage modulation as

$$
\hat{n}=\frac{c_{\mu}^{n}}{q} \hat{V}
$$

Here the quantity

$$
c_{\mu}^{n}=q \frac{\partial n}{\partial V}=\frac{q^{2} n}{k_{B} T}
$$

is a chemical capacitance (per unit volume) of the freely moving ions intercalated in the lattice. Similarly we define a chemical capacitance for the lithium ions in LiX sites (related to that previously introduced in Eq. (1))

$$
c_{\mu}^{c}=q \frac{\partial c}{\partial V}=\frac{q^{2} c\left(N_{1}-c\right)}{N_{1} k_{B} T}
$$

It should be observed that the chemical capacitance is correlated to the derivative of the voltagecomposition curves. This capacitance is not related to positive-negative charge separation at an interface.[48] In the simple model outlined here $c_{\mu}$ is given by the entropy of ions in equivalent cites. In general $c_{\mu}$ relates to the dependence of the chemical potential on the concentration and depends on any chemical force over the ions in the solid phase.

Modulation of the voltage with angular frequency $\omega$ changes the free ion density which produces a change of the reacted density as $c(t)=\bar{c}+\hat{c}$. By a Laplace transform and expanding to first order, we obtain from Eq. (7) the relationship

$$
\hat{c}=\frac{k_{1}\left(N_{1}-\bar{c}\right)}{\omega_{c r}+i \omega} \hat{n}
$$

where 


$$
\omega_{c r}=k_{1} \bar{n}+k_{2} N_{0}
$$

is the characteristic kinetic frequency of the conversion reaction previously introduced in Eq. (2). On the other hand Eq. (8) gives

$$
\left[i \omega+k_{1}\left(N_{1}-c\right)\right] \hat{n}=\left(k_{1} \bar{n}-k_{2} N_{0}\right) \hat{c}+\frac{\hat{J}_{n}}{L}
$$

Inserting Eq. (11) and (102) in Eq. (16) we arrive at the result

$$
\left(C_{\mu}^{n} i \omega+\frac{C_{\mu}^{c} i \omega}{1+i \omega / \omega_{c r}}\right) \hat{V}=\hat{j}
$$

Here $C_{\mu}^{n}=L c_{\mu}^{n}$ and $C_{\mu}^{c}=L c_{\mu}^{c}$ are chemical capacitances per unit area. The admittance is defined as $Y=\hat{j} / \hat{V}$, therefore we have

$$
Y=C_{\mu}^{n} i \omega+Z_{c r}^{-1}
$$

Where

$$
Z_{c r}=R_{c r}+\frac{1}{C_{\mu}^{c} i \omega}
$$

Here we have introduced the resistance of the conversion reaction $R_{c r}$ given by

$$
\begin{aligned}
& R_{c r}{ }^{-1}=C_{\mu}^{c} \omega_{c r} \\
& =\frac{L q^{2}}{N_{1} k_{B} T} \bar{c}\left(N_{1}-\bar{c}\right)\left(k_{1} \bar{n}+k_{2} N_{0}\right)
\end{aligned}
$$

The impedance model in Eq. (18) corresponds to the previously introduced equivalent circuit in Figure 4c.

\section{References}

1. Tarascon, J.M. and M. Armand, Issues and challenges facing rechargeable lithium batteries. Nature, 2001. 414(6861): p. 359-367.

2. Zhu, J., et al., Nanohybridization of ferrocene clusters and reduced graphene oxides with enhanced lithium storage capability. Chemical Communications, 2011. 47(37): p. 10383-10385.

3. Wang, Z., et al., [small alpha]-Fe2O3 nanotubes with superior lithium storage capability. Chemical Communications, 2011. 47(28): p. 8061-8063.

4. Wang, B., et al., Quasiemulsion-Templated Formation of $\alpha$-Fe2O3 Hollow Spheres with Enhanced Lithium Storage Properties. Journal of the American Chemical Society, 2011. 133(43): p. 1714617148.

5. France Patent 1490725, 1966.

6. Guan, D., C. Cai, and Y. Wang, Amorphous and Crystalline TiO2 Nanotube Arrays for Enhanced LiIon Intercalation Properties. Journal of Nanoscience and Nanotechnology, 2011. 11(4): p. 36413650. 
7. Li, X., et al., Batteries: Tin Oxide with Controlled Morphology and Crystallinity by Atomic Layer Deposition onto Graphene Nanosheets for Enhanced Lithium Storage (Adv. Funct. Mater. 8/2012). Advanced Functional Materials, 2012. 22(8): p. 1646-1646.

8. Wang, X.-L., et al., Amorphous Hierarchical Porous GeOx as High-Capacity Anodes for Li Ion Batteries with Very Long Cycling Life. Journal of the American Chemical Society, 2011. 133(51): p. 20692-20695.

9. $\quad \mathrm{Ku}$, J.H., et al., Reversible Lithium Storage with High Mobility at Structural Defects in Amorphous Molybdenum Dioxide Electrode. Advanced Functional Materials, 2012: p. n/a-n/a.

10. Controlled synthesis and gas-sensing properties of hollow sea urchin-like $\alpha$-Fe2O3 nanostructures and $\alpha$-Fe2O3 nanocubes. Sensors and Actuators B Chemical, 2009. 141(2): p. 381.

11. Flexible morphology-controlled synthesis of mesoporous hierarchical $\alpha$-Fe2O3 architectures and their gas-sensing properties. CrystEngComm, 2011. 13(3): p. 806.

12. Synthesis of spindle-shaped $\alpha-\mathrm{FeOOH}$ and $\alpha-\mathrm{Fe} 2 \mathrm{O} 3$ nanocrystals. Crystal Research and Technology, 2011: p. n/a.

13. Tabuchi, T., et al., Surface reaction of $\mathrm{B}-\mathrm{FeOOH}$ film negative electrode for lithium-ion cells. Journal of Power Sources, 2009. 191(2): p. 636-639.

14. Zhu, J., et al., Hierarchical hollow spheres composed of ultrathin Fe2O3 nanosheets for lithium storage and photocatalytic water oxidation. Energy \& Environmental Science, 2013. 6(3): p. 987993.

15. Zhang, W., et al., $\mathrm{Fe} 2 \mathrm{O} 3$ nanocluster-decorated graphene as $\mathrm{O} 2$ electrode for high energy Li-O2 batteries. RSC Advances, 2012. 2(22): p. 8508-8514.

16. Reddy, M.V., et al., $\alpha$-Fe2O3 Nanoflakes as an Anode Material for Li-lon Batteries. Advanced Functional Materials, 2007. 17(15): p. 2792-2799.

17. Xu, C., et al., Controlled Soft-Template Synthesis of Ultrathin C@FeS Nanosheets with High-LiStorage Performance. ACS Nano, 2012. 6(6): p. 4713-4721.

18. Sun, Z., X. Feng, and W. Hou, Morphology-controlled synthesis of $\alpha$-FeOOH and its derivatives. Nanotechnology, 2007. 18(45): p. 455607.

19. Mansour, A.N. and R.A. Brizzolara, Characterization of the Surface of alpha-FeOOH Powder by XPS. Surface Science Spectra, 1996. 4(4): p. 357-362.

20. Zhang, C., et al., Synthesis of hexagonal-symmetry [small alpha]-iron oxyhydroxide crystals using reduced graphene oxide as a surfactant and their Li storage properties. CrystEngComm, 2012. 14(1): p. 147-153.

21. Wang, Y., A. Muramatsu, and T. Sugimoto, FTIR analysis of well-defined $\alpha$-Fe2O3 particles. Colloids and Surfaces A: Physicochemical and Engineering Aspects, 1998. 134(3): p. 281-297.

22. Sing; K.S.W., et al., Reporting physisorption data for gas/solid systems with special reference to the determination of surface area and porosity. Pure Appl. Chem., 1985. 57(4): p. 603-619.

23. Lou, X., X. Wu, and Y. Zhang, Goethite nanorods as anode electrode materials for rechargeable Li-ion batteries. Electrochemistry Communications, 2009. 11(8): p. 1696-1699.

24. Pinson, M.B. and M.Z. Bazant, Theory of SEI Formation in Rechargeable Batteries: Capacity Fade, Accelerated Aging and Lifetime Prediction. Journal of The Electrochemical Society, 2012. 160(2): p. A243-A250.

25. Arora, P., R.E. White, and M. Doyle, Capacity Fade Mechanisms and Side Reactions in Lithium-lon Batteries. Journal of The Electrochemical Society, 1998. 145(10): p. 3647-3667.

26. Sikha, G., B.N. Popov, and R.E. White, Effect of Porosity on the Capacity Fade of a Lithium-Ion Battery: Theory. Journal of The Electrochemical Society, 2004. 151(7): p. A1104-A1114.

27. Guerrero, A., et al., Recombination in Organic Bulk Heterojunction Solar Cells: Small Dependence of Interfacial Charge Transfer Kinetics on Fullerene Affinity. J. Phys. Chem. Lett., 2012. 3: p. 1386-1392. 
28. Wang, Z., et al., Amorphous CoSnO3@C nanoboxes with superior lithium storage capability. Energy \& Environmental Science, 2013. 6(1): p. 87-91.

29. Xiang, J.Y., et al., Electrochemical Impedance Analysis of a Hierarchical CuO Electrode Composed of Self-Assembled Nanoplates. The Journal of Physical Chemistry C, 2011. 115(5): p. 2505-2513.

30. Ruffo, R., et al., Impedance Analysis of Silicon Nanowire Lithium Ion Battery Anodes. The Journal of Physical Chemistry C, 2009. 113(26): p. 11390-11398.

31. Wang, C., A.J. Appleby, and F.E. Little, Electrochemical impedance study of initial lithium ion intercalation into graphite powders. Electrochimica Acta, 2001. 46(12): p. 1793-1813.

32. Wang, C. and J. Hong, lonic/Electronic Conducting Characteristics of LiFePO4 Cathode Materials: The Determining Factors for High Rate Performance. Electrochemical and Solid-State Letters, 2007. 10(3): p. A65-A69.

33. Garcia-Belmonte, G., J. García-Cañadas, and J. Bisquert, Correlation between Volume Change and Cell Voltage Variation with Composition for Lithium Intercalated Amorphous Films. J. Phys. Chem. B, 2006. 110: p. 4514-4518.

34. Pitarch, A., et al., Electrochemical Impedance Spectra for the Complete Equivalent Circuit of Diffusion and Reaction under Steady-State Recombination Current. Phys. Chem. Chem. Phys., 2004. 6: p. 2983-2988.

35. Jamnik, J. and J. Maier, Generalised equivalent circuits for mass and charge transport: chemical capacitance and its implications. PhysChemChemPhys, 2001. 3: p. 1668-1678

36. Levi, M.D. and D. Aurbach, Impedance of a Single Intercalation Particle and of NonHomogeneous, Multilayered Porous Composite Electrodes for Li-ion Batteries. J. Phys. Chem. B, 2004. 108: p. 11693-11703.

37. Meyers, J.P., et al., The Impedance Response of a Porous Electrode Composed of Intercalation Particles J. Electrochem. Soc., 2000. 147: p. 2930-2940.

38. Garcia-Belmonte, G., et al., Relaxation processes in the coloration of amorphous WO3 thin films studied by combined impedance and electro-optical measurements. Journal of Applied Physics, 2004. 96: p. 853-859.

39. Cabana, J., et al., Beyond Intercalation-Based Li-Ion Batteries: The State of the Art and Challenges of Electrode Materials Reacting Through Conversion Reactions Advanced Energy Materials, 2010. 22: p. E170-E192.

40. Khatib, R., et al., Origin of the Voltage Hysteresis in the CoP Conversion Material for Li-lon Batteries. J. Phys. Chem. C, 2013. 117: p. 837-849.

41. Ponrouch, A. and M.R. Palacin, Optimisation of performance through electrode formulation in conversion materials for lithium ion batteries: Co3O4 as a case example. Journal of Power Sources, 2012. 212(0): p. 233-246.

42. Khatib, R., et al., Origin of the Voltage Hysteresis in the CoP Conversion Material for Li-lon Batteries. The Journal of Physical Chemistry C, 2013. 117: p. 837-849.

43. McKinnon, W.R. and R.R. Haering, Physical mechanisms of intercalation, in Modern Aspects of Electrochemistry, R.E. White, J.O.M. Bockris, and B.E. Conway, Editors. 1983, Plenum Press: New York. p. 235-304.

44. Gao, Y., J.N. Reimers, and J.R. Dahn, Changes in the voltage profile of li/Li1+xMn2-xO4 cells as a fuction of $x$. Physical Review B, 1996. 54: p. 3878-3883.

45. Bisquert, J. and V.S. Vikhrenko, Analysis of the kinetics of ion intercalation. Two state model describing the cpupling of solid state ion diffusion and ion binding processes. Electrochimica Acta, 2002. 47: p. 3977-3988.

46. Levi, M.D., et al., a comparative study of Mg2+ and Li+ ion insertions into the Mo658 Chevrel phase using electrochemical impedance spectroscopy. Journal of Electroanalytical Chemistry, 2004. 569: p. 211-223. 
47. Levi, M.D. and D. Aurbach, Distinction between Energetic Inhomogeneity and Geometric NonUniformity of Ion Insertion Electrodes Based on Complex Impedance and Complex Capacitance Analysis. The Journal of Physical Chemistry B, 2005. 109: p. 2763-2773.

48. Bisquert, J., Chemical Capacitance of Nanostructured Semiconductors: its Origin and Significance for Heterogeneous Solar Cells. Phys. Chem. Chem. Phys., 2003. 5: p. 5360-5364.

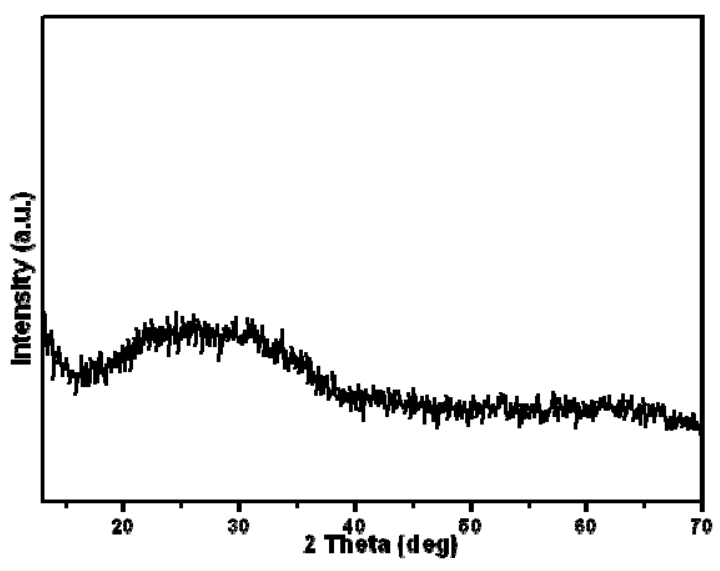

Figure 1. XRD pattern of the as-synthesized powder
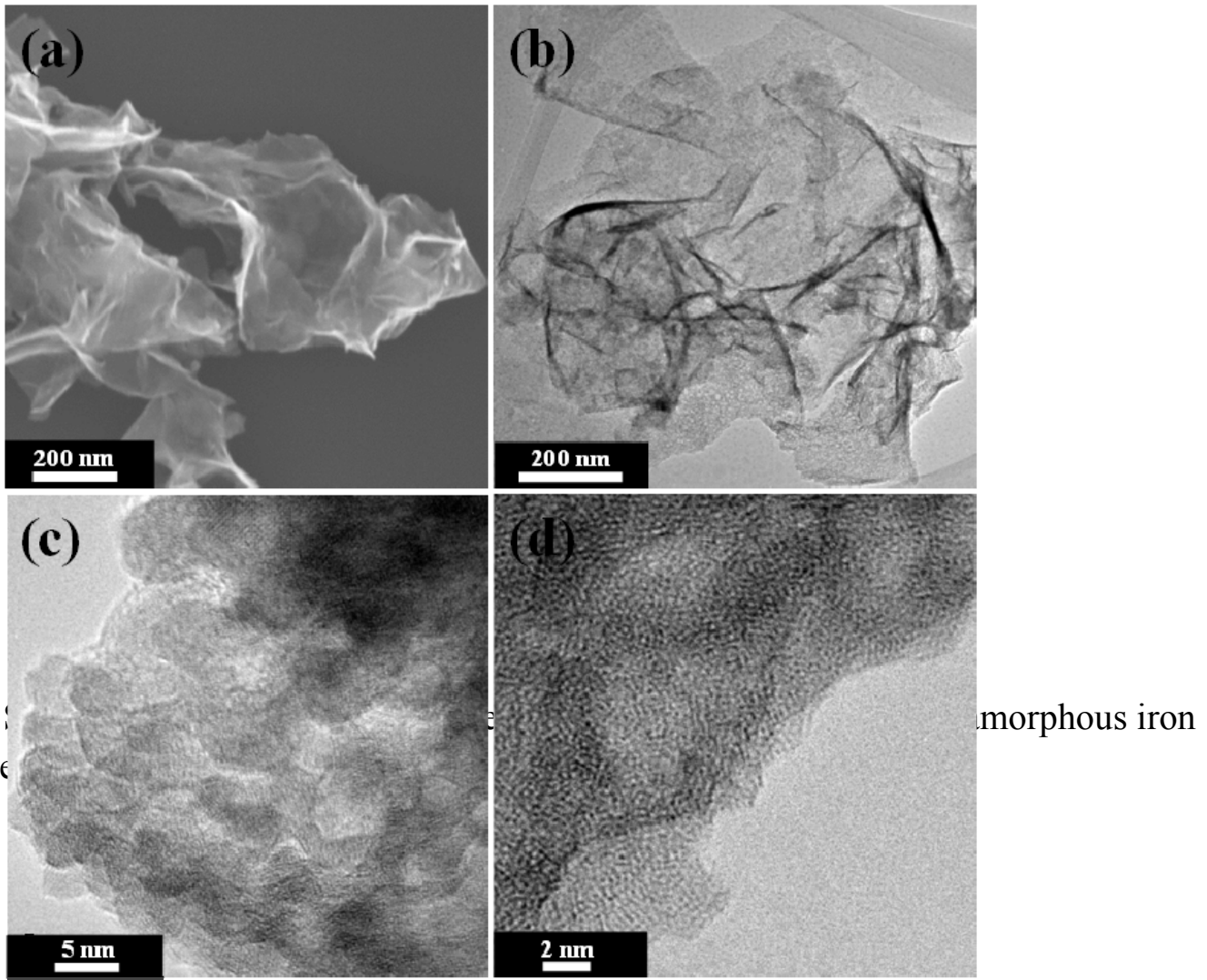

Figure 3. (a)

(c) oxyhydroxide 
Figure 2. (a) SEM image; (b) TEM image; (c) and (d) HRTEM image of the amorphous iron oxyhydroxide nanosheet
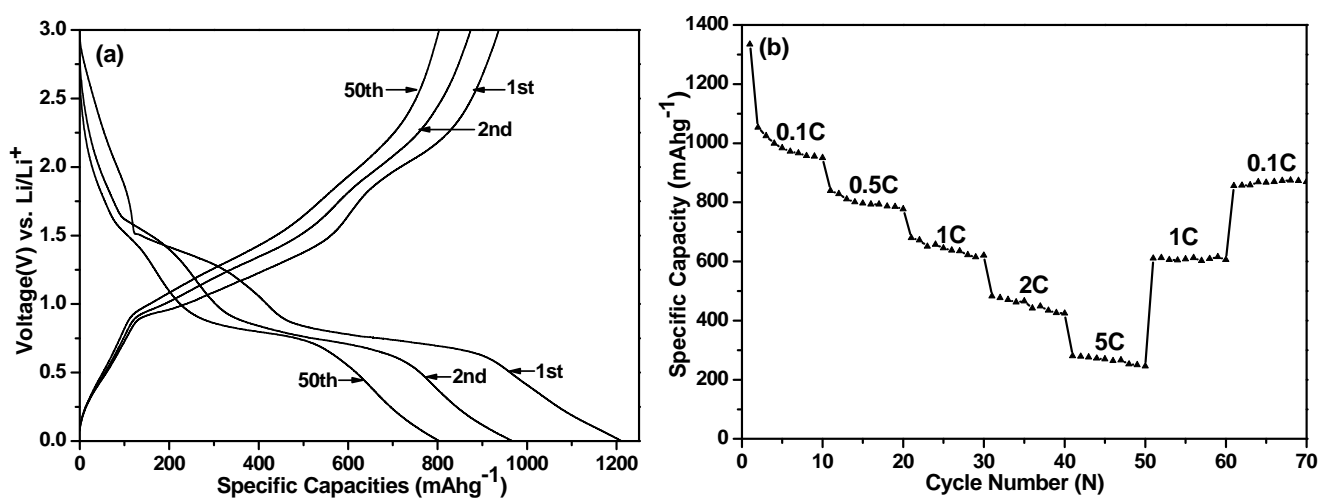

Figure 3. (a) Charge-discharge voltage profiles of the $1^{\text {st }}, 2^{\text {nd }}$ and $50^{\text {th }}$ cycle; (b) cycling performance at different C-rates 
(a)

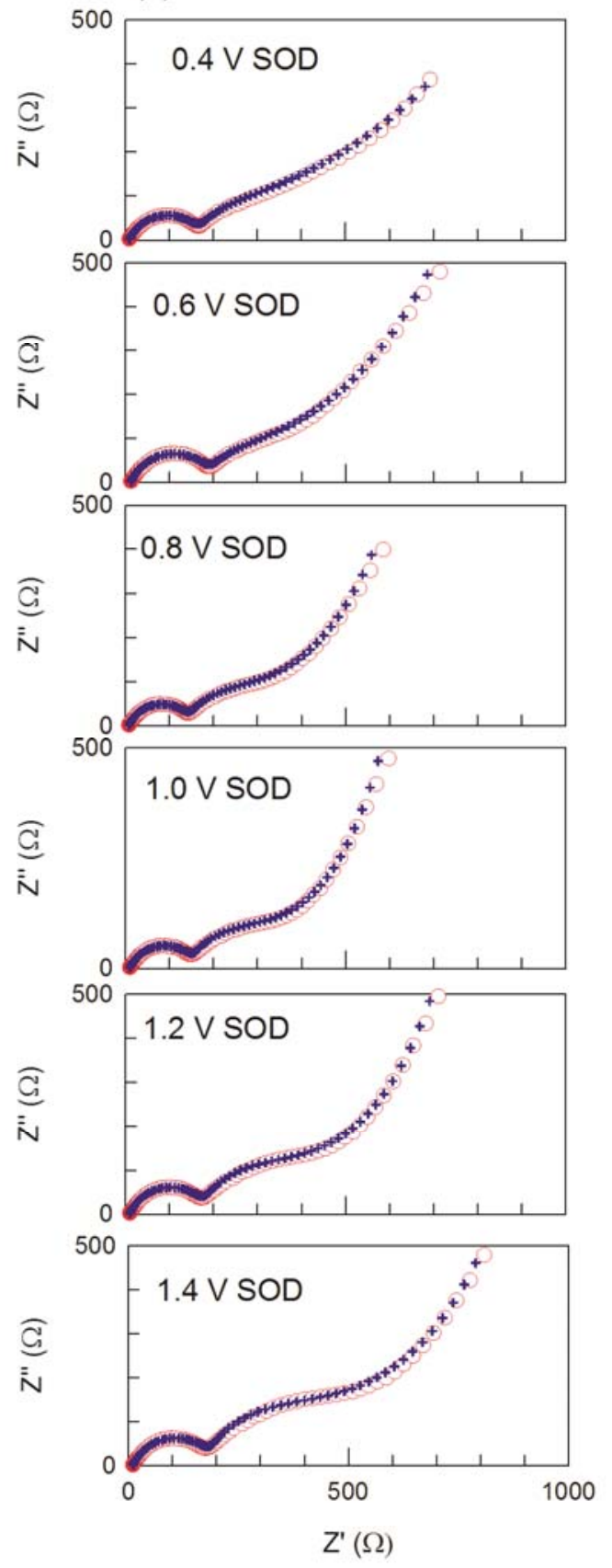

(b)
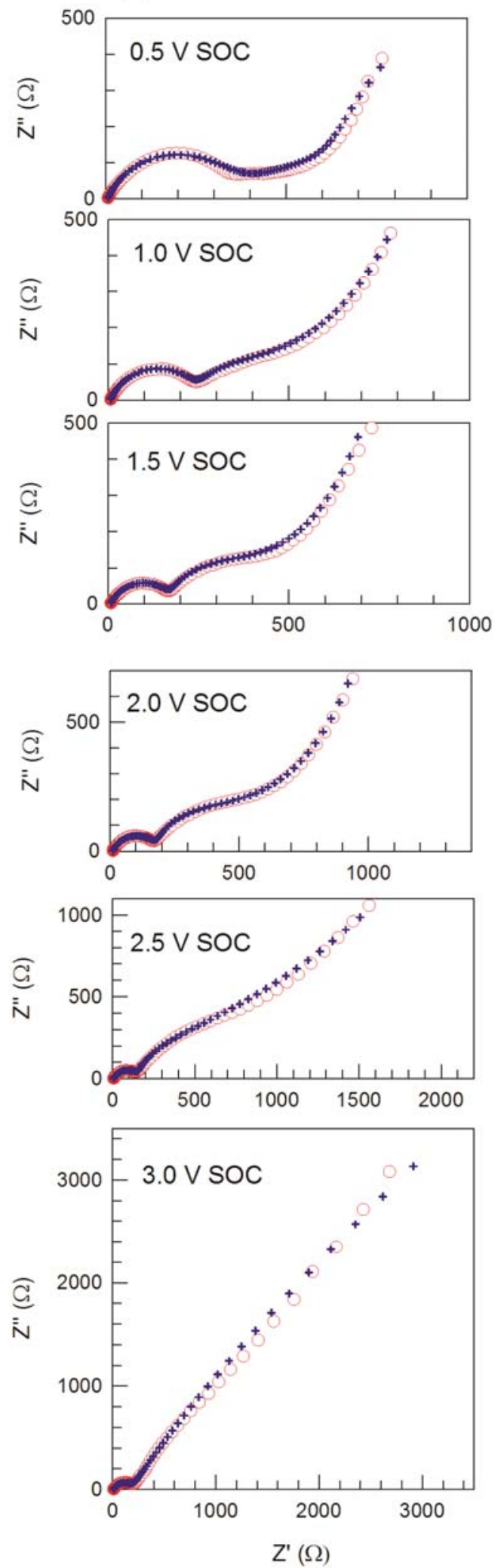


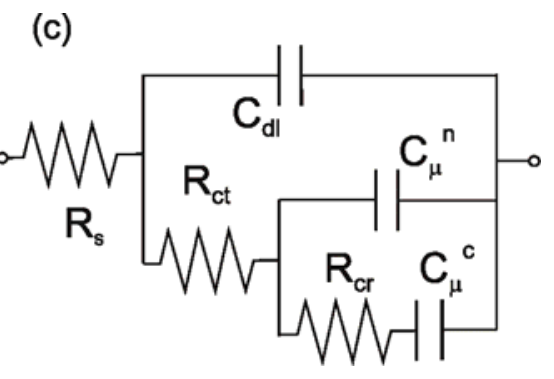

Figure 4 Impedance response measured at different voltages corresponding either to (a) discharge regime SOD or (b) charge regime SOC. Experimental data (dot) and fits (cross) are displayed for comparison. (c)

Equivalent circuit employed in fitting which includes double layer capacitance, electrolyte/electrode charge transfer resistance, and conversion reaction circuit elements. 

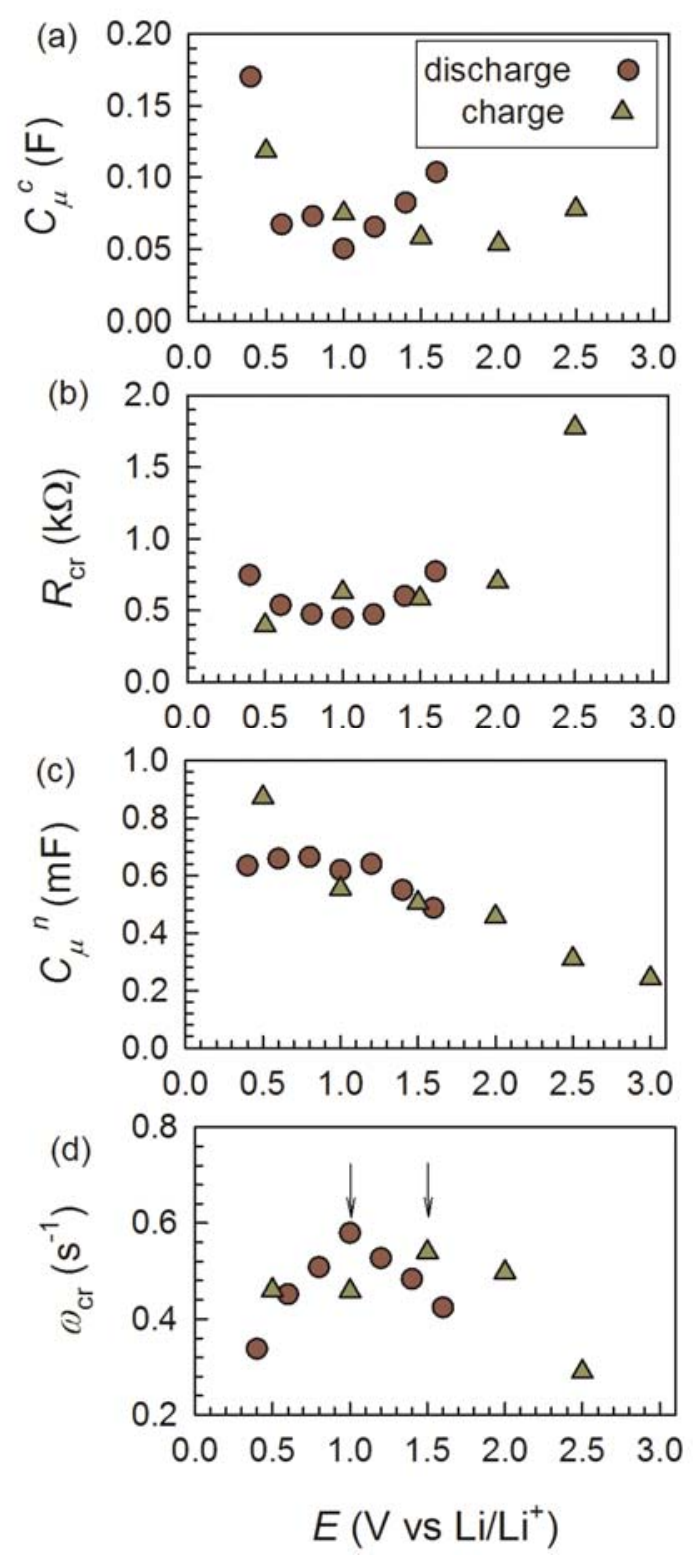

Figure 5 Parameters extracted from fitting using the equivalent circuit in Figure 4c. (a) Chemical capacitance corresponding to reacted Li ions, (b) conversion reaction resistance, (c) chemical capacitance corresponding to Li moving ions, and (d) conversion reaction response frequency. 